\title{
Quality Improvement in Color Image Compression using New FDCT and FIDCT
}

\author{
G.J. Joyce Mary, PhD \\ Research Guide \\ Department of Computer Science and Engineering \\ PRIST University, Vallam, Thanjavur \\ Tamilnadu, India
}

\author{
A. Ramachandran \\ Ph.D Research Scholar and Assistant Professor \\ Department of Computer Science and Engineering \\ PRIST University, Vallam, Thanjavur, India \\ Srinivasan Engineering College Tamilnadu, India
}

\begin{abstract}
The image compression methods aim to compress color image while ensuring that noise removal compression technique produce the good quality level images. The storage of images is becoming difficult with number of images growing to million and billions. Hence the image compression becoming absolute necessity in computing field. The Discrete Cosine Transform is widely used in image compression technique. Redundant information in an image needs to be eliminated by adopting intelligent method. In the recent research attempts better quality of compression is observed with the use of Discrete Cosine Transform (DCT) and Quantization. In this paper, the proposed system efficient image encoder removes the sinusoidal noise from original image by Butterworth Band Reject Filter (BBRF) algorithm in FDCT color image compression. The objective of this color image compression scheme is efficiently noise removal image, calculate the Compression Ratio(CR), Peak Signal to Noise Ratio (PSNR) Mean Square Error (MSE) by changing the FDCT level and using Fine Inverse Discrete Cosine Transform (F-IDCT) factor while preserving the quality of reconstructed image. Experimentation has been carried out on different image formats successfully. The proposed system achieved a good compression ratio and considerable test application for the quality of the reconstructed color image.
\end{abstract}

\section{Keywords}

FDCT, FIDCT, PSNR, MSE, Sinusoidal Noise, BBRF, Image Compression.

\section{INTRODUCTION}

Recently, a new image compression technique for sinusoidal noise removal image compression method is popular. Image compression is a part of data compression. Due to size of digital images they engage more space in storage. Also they take more bandwidth and time for getting upload \& download from internet. The image compression will become fruitful when the superiority of image gets maintained. Wavelets give the better performance for maintaining image quality in image compression. Image compression can be done by any of two types are Lossy Compression and Lossless Compression. In Lossy Compression which is used in fewer amounts of data in loss of clearness of images are allowed also human eyes will not be able to detect the difference between original and compressed images. In case lossless type of image compression the loss of clarity of images is not allowed [1].

The wavelet is mathematical tools for mining information from various types of data which is also includes images. So they can be "mixed up" using actions of reversing multiplying, summing which is also called as convolution. These wavelets are becoming very useful for huge number of applications like fingerprint application, palm print application, Steganography [2], Iris recognition. The wavelets exploited largely and also grow to be important in the fields of image compression, analyses of Electrocardiogram (ECG), Deoxyribonucleic Acid (DNA), Face and speech recognition, computer graphics. It also helps for multi resolution analysis. They have been used in reduction of noise from data, [3] compression of multispectral images and compression of SAR images. Compression is reduces the storage requirements, transmission time and bandwidth, which makes the data management more effective and efficient [4], [5], [6]. The Process of encoding all DCT coefficients and transmitting them requires huge bandwidth and serve no purpose since most of the coefficients are zeros [12].

By analyzing this, proposed system an intelligent and Butterworth noise removal technique for selection of significant coefficients by eliminating redundancy. In Filter based sinusoidal noise removal computed for each image will adaptive select the significant coefficients by reducing noise and MSE and FDCT factor while preserving the quality of information in reconstruct image.

\section{RELATED WORKS}

\subsection{Colorization-Based Compression Techniques [13]}

The main function of colorization based coding is an extraction of the Representative Pixel (RP). Previous colorization based coding methods use an iterative approach to extract the RP. In these approaches a prior temporary set of $\mathrm{RP}$ is usually selected. This is a prior selection is manual and causes a redundant or insufficient set of RP. Therefore, redundant RP have to be eliminated and required RP have to additionally extract by additional RP elimination or extraction methods.

\subsection{DCT and IDCT [7]}

Transform coding systems based on a variety of discrete transform have been constructed extensively. The choice of particular transform in a given application depends on the amount of reconstruction error that can be tolerated and the computational resource available. Compression is achieved during the quantization of the transformed coefficient value.

Consider an image $f(x, y)$ of size NxN whose forward, discrete transform $\mathrm{T}(\mathrm{u}, \mathrm{v})$ can be expressed in terms of the general relation 


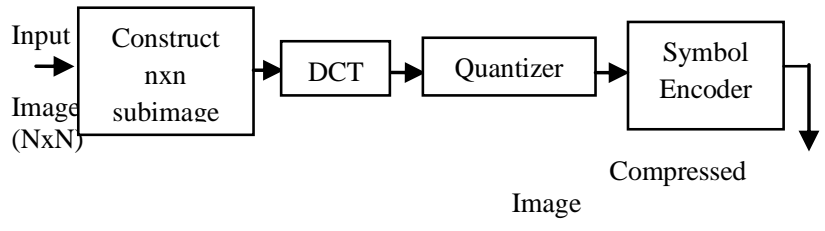

Fig 1: Image Compression System

$$
T(u, v)=\sum_{u=0}^{N-1} \sum_{\substack{v=0 \\ \text { Eq. } 1}}^{N-1} f(x, y) g(x, y, u, v) \ldots
$$

for $\mathrm{u}, \mathrm{v}=0,1,2 \ldots \mathrm{N}-1$. Given $\mathrm{T}(\mathrm{u}, \mathrm{v}), \mathrm{f}(\mathrm{x}, \mathrm{y})$ similarly can be obtained using the generalized inverse discrete transform.

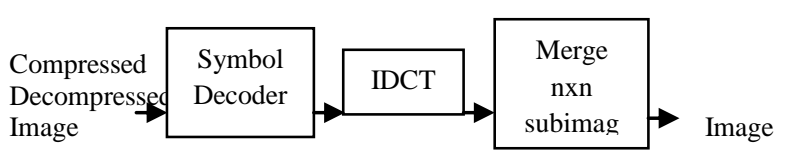

Fig 2: Image Decompression System

$$
f(x, y)=\sum_{x=0}^{N-1} \sum_{\substack{\text { Eq. } 2 \\ N-1}}^{-1} T(x, y) h(x, y, u, v) \ldots
$$

for $\mathrm{x}, \mathrm{y}=0,1,2 . . \mathrm{N}-1$. In this equation, $\mathrm{g}(\mathrm{x}, \mathrm{y}, \mathrm{u}, \mathrm{v})$ and $\mathrm{h}(\mathrm{x}, \mathrm{y}, \mathrm{u}, \mathrm{v})$ are called forward and inverse transform.

\subsection{MSE, RMSE and PSNR [7]}

The Mean Squared Error (MSE), Root Mean Squared Error (RMSE) and Peak Signal to Noise Ratio (PSNR) are calculated using equation - (3),(4) and (5) by following formula respectively.

$$
M S E=\frac{1}{m n} \sum_{i} \sum_{j}[M(i, j)-R(i, j)]^{2} \quad \ldots \text { Eq. } 3
$$

Where $\mathrm{M}(\mathrm{i}, \mathrm{j})$ is original image and $\mathrm{R}(\mathrm{i}, \mathrm{j})$ is reconstructed image respectively.

$$
\begin{aligned}
& R M S E=\sqrt{M S E} \\
& P S N R=20 * \log _{10} \frac{255}{R M S E}(d B)
\end{aligned}
$$

\section{PROPOSED WORK}

In this paper the system proposed new compression technique called sinusoidal noise removal in FDCT image compression. As an initial requirement like Fine Discrete Cosine Transform, image segmentation method, Sinusoidal noise removal mechanism, PSNR, MSE and reconstruct of F-IDFT methods are discussed following below.

\subsection{System Design}

Figure 3 shows the architectural diagram of the system of proposed method. The details of the components are described in the following subsections. The image encoder removes the sinusoidal noise from the original image by Butterworth Band Reject Filter Algorithm. The resultant image is implementing to FDCT and quantization technique. This image is applied to F-IDCT decoding image compression technique. This technique will improve the color image quality. The system process is implementing to FDCT in the processing term in encoding. The transmission process of encoding image is converted in to decoding procedure. The decode process is applied to FIDCT method. This is ready to implement to Dequantization and FIDCT technique. Finally proposed system getting a good quality of noise removed reconstructed image.
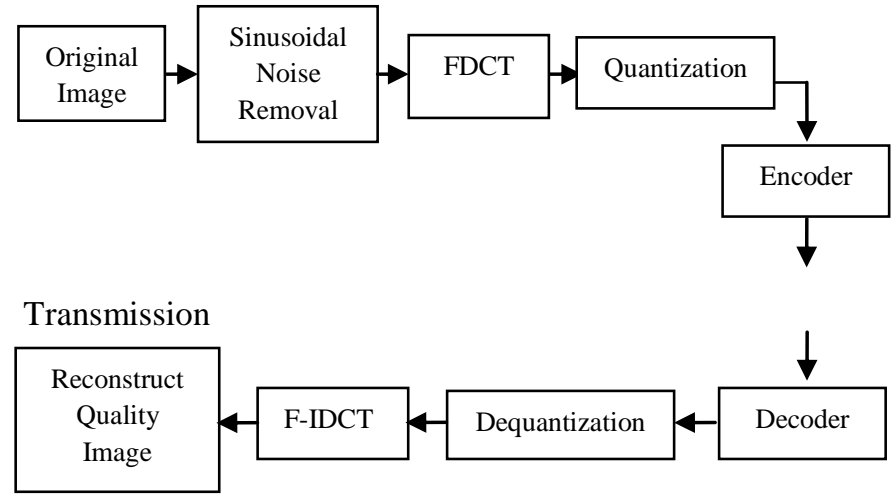

Fig 3: System Design of proposed image compression

\subsection{Butterworth Band Reject Filtering Algorithm}

The more specialized band reject filter is used as tool for sinusoidal noise reduction or removal. One of the principal applications of band reject filtering is for noise removal in applications where the general location of the noise components in the frequency domain approximately known. A good example is an image corrupted by additive periodic noise that can be approximated as tow-dimensional sinusoidal functions. The Fourier transform of a pure sine is a pair of impulses. One impulse is located at the frequency of the sine, and other at the mirror image of that point. The impulses are both imaginary (the real part of the Fourier transform of a sine is zero) and are complex conjugates of each other. This Butterworth Band Reject Filter with the appropriate radius and width to enclose completely the noise impulse. Since it is desirable in general to remove as little as possible from transform, sharp, narrow filters are common in band reject filtering. The improvement is quite evident.

Band Reject Filter removes or attenuates a band of frequencies about the origin of the Fourier transform. A Band Reject filter is given by the expression equation5 respectively [14]

$$
H(u, v)=\left\{\begin{array}{l}
1 \text { if } \mathrm{D}(\mathrm{u}, \mathrm{v})<\mathrm{D}_{0}-\mathrm{W} / 2 \\
0 \text { if } \mathrm{D}_{0}-\mathrm{W} / 2<=\mathrm{D}_{0}+\mathrm{W} / 2 \\
1 \text { if } \mathrm{D}(\mathrm{u}, \mathrm{v}),>\mathrm{D}_{0}+\mathrm{W} / 2
\end{array} \ldots \text {... } 6\right.
$$

Where $\mathrm{D}(\mathrm{u}, \mathrm{v})$ is the distance from the origin of the centered frequency rectangle as given by the equation, $\mathrm{W}$ is the width of the band, and $\mathrm{D}_{0}$ is its radial center.

Similarly, a Butterworth Band Reject Filter of order $\mathrm{n}$ is given by the expression equation6 respectively. 
$\mathrm{H}(\mathrm{u}, \mathrm{v})=$

...Eq.7

\subsection{Newly Proposed FDCT and FIDCT}

The Fine Cosine Transform has found wide application in transform image coding. The efficacy of FDCT on images is discussed here. System proposed the new 2D FDCT and FIDCT. The newly proposed method is explain given by

FDCT function is defined given by

$F D(u, v)=\frac{2}{n} \alpha(u) \alpha(v) \sum_{x=0}^{M-1} \sum_{y=0}^{N-1} F I(x, y) P * Q$

Where

$$
\begin{aligned}
\alpha(u) & =\sqrt{\frac{1}{M}} \text { for } \mathrm{u}=0,1,2 \ldots \mathrm{N}-1 \\
\alpha(v) & =\sqrt{\frac{1}{N}} \text { for } \mathrm{v}=0,1,2 \ldots \mathrm{N}-1 \\
P & =\cos \left[\sqrt{\frac{(2 x+1) u \pi}{2 M}}\right]^{2} \\
Q & =\cos \left[\sqrt{\frac{(2 y+1) v \pi}{2 N}}\right]^{2}
\end{aligned}
$$

FD - Fine Discrete Transform

$\mathrm{FD}(\mathrm{u}, \mathrm{v})$ - FD Function

$\mathrm{u}$, and $\mathrm{v}-$ Frequency variable

$\mathrm{M}$ and $\mathrm{N}$ - Transform function

The inverse transform is defined as given equation8 respectively

$$
F I(x, y)=\frac{2}{n} \alpha(u) \alpha(v) \sum_{u=0}^{M-1} \quad \sum_{v=0}^{N-1} \quad F D(u, v) P * Q
$$

Where

$$
\begin{gathered}
\alpha(u)=\sqrt{\frac{1}{M}} \text { for } \mathrm{u}=0,1,2 \ldots \mathrm{N}-1 \\
\alpha(v)=\sqrt{\frac{1}{N}} \text { for } \mathrm{v}=0,1,2 \ldots \mathrm{N}-1 \\
P=\cos \left[\sqrt{\frac{(2 x+1) u \pi}{2 M}}\right]^{2} \\
Q=\cos \left[\sqrt{\frac{(2 y+1) v \pi}{2 N}}\right]^{2}
\end{gathered}
$$

FI - Fine Inverse Transform

$\mathrm{FI}(\mathrm{x}, \mathrm{y})$ - Inverse Function

$\mathrm{x}$ and $\mathrm{y}-$ Image Variables

$\mathrm{M}$ and $\mathrm{N}$ - Transform Function

The 2-D basis functions can be generated by multiplying the horizontally with vertically oriented set of the same functions. The basic functions image pixel for $\mathrm{M}=8$ and $N=8$. It can be noted that the basic functions exhibit a progressive increase in frequency and image variable both in the vertical and horizontal direction. The basic transformation functions of results from multiplication of FDC component in with its transpose images. Hence, this function assumes a constant value and is referred to as the FDCT coefficient.

\subsection{Proposed Methodology}

The image is broken into $8 \times 8$ block of pixels. Each block of pixels used to image compression. In this kind of broken block of pixels is very difficult. Block of pixels is using to produce high quality of image compression. Each and every block is information about the picture. Splitting of picture block used in compression mechanism and also merging of picture block is used in decompression mechanism. Each dividing blocks are the subdivision of images. The sample FDCT method for described in figure 4. Working from left to right, top to bottom the FDCT is applied to each block. The FDCT fully used for compression standards. In this method produce high quality compression. It is supported to Sinusoidal Noise Removal steps. Each block is compressed through quantization. The array of compressed block that constitutes the image is stored in a drastically reduced amount of space. When desired, the image is reconstructed through decompression, a process that uses the Fine Inverse Discrete Cosine Transform (FIDCT). It is the inverse of FDCT. So that decompression is important one. FIDCT is very successful manner of reconstruct the color image.

\section{FDCT:}

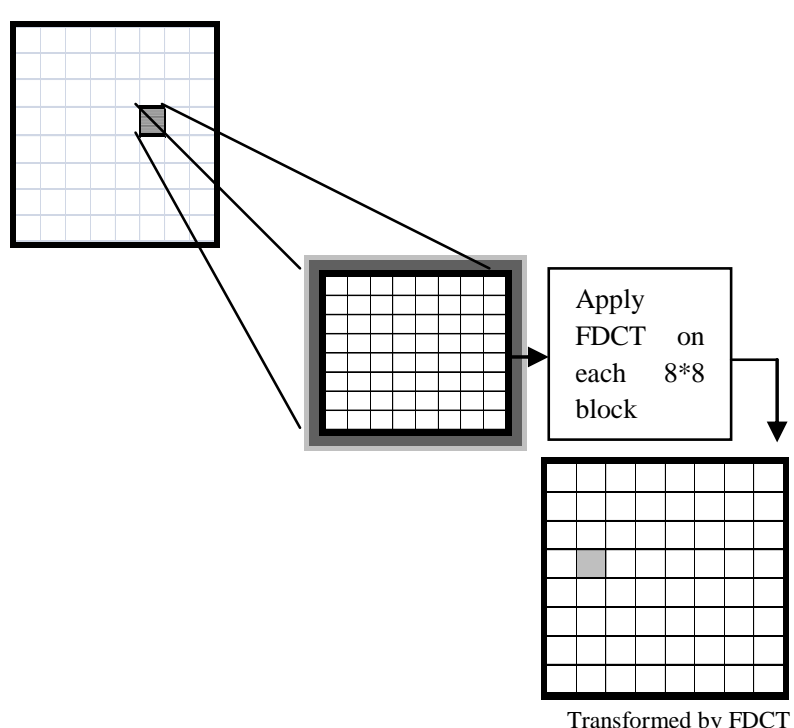

Fig 4: FDCT Code Form 
The plane is divided into blocks of $8 * 8$ sizes and FDCT applied to each block. The FDCT coefficients are compressed through the quantization process using quantize. The FDCT coefficients values become zero. The scanning mechanism is zig zag which means left to right. In this technique which is used to convert matrix into array formats. The encoding is used to convert coefficients into binary value and the same is transmitted.

\section{FIDCT:}

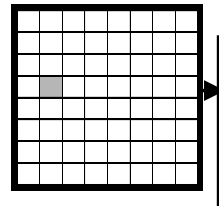

Inverse
FDCT on
each $8 * 8$
block

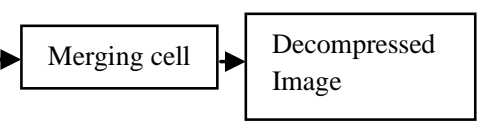

image

\section{Fig 5: FDCT Code Form}

The binary value are transmitted and decoded at the receiving end. The $8 * 8$ size is merged FDCT is applied to each block. The FIDCT coefficient are decompressed obtain the reconstructed image.

Table 1. Description of Proposed Method

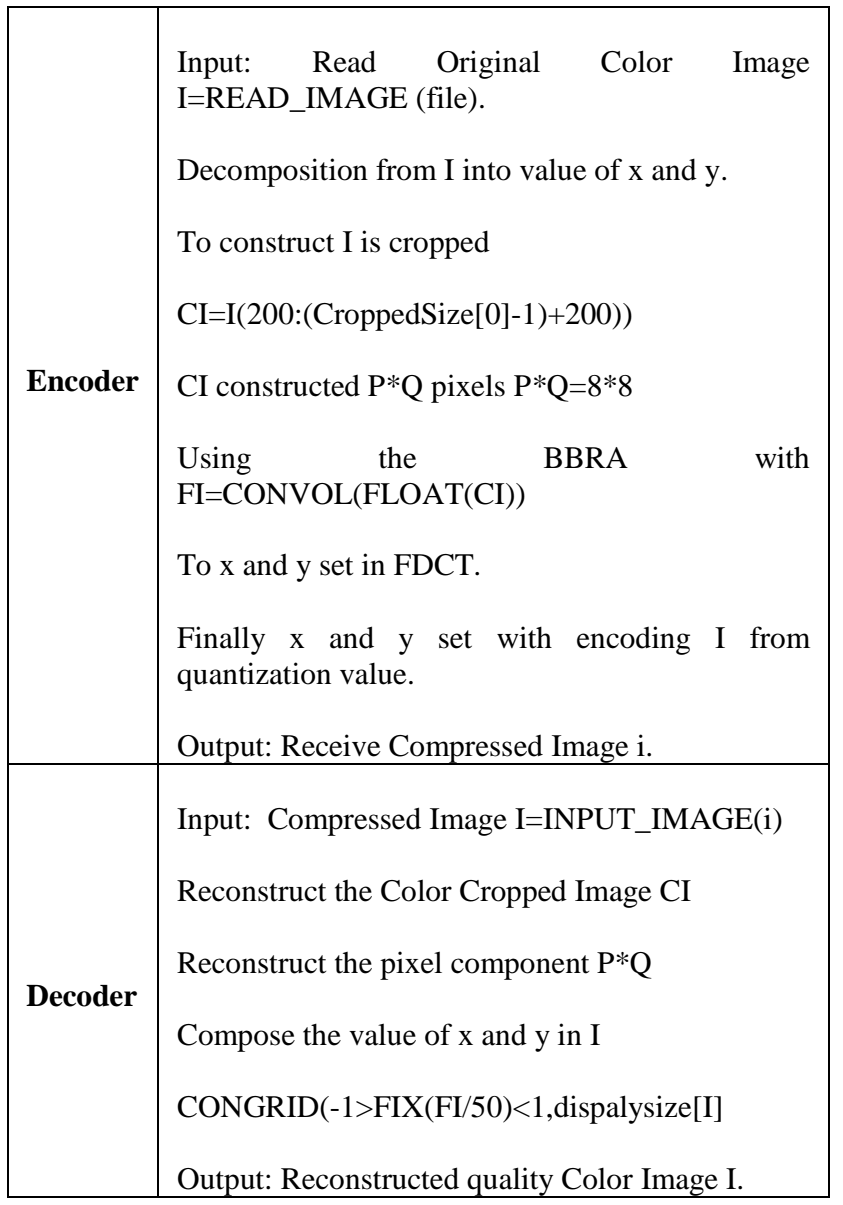

\subsection{Quantizer [10]}

A quantizer simply reduces the number of bits needed to store the transformed coefficients by reducing the precision of those values. Since this is a many-to-one mapping, it is a lossy process and is the main source of compression in an encoder. Quantization can be performed on each individual coefficient, which is known as Scalar Quantization (SQ). Quantization can also be performed on a group of coefficients together, and this is known as Vector Quantization (VQ).

The quantizer sub-block utilizes the fact that the human eye is unable to perceive some visual information in an image. Such information is deemed redundant and can be discarded without introducing noticeable visual artifacts. Such redundancy is referred to as psychovisual redundancy. This idea can be extended to low bitrate receivers which, due to their stringent bandwidth requirements, might sacrifice visual quality in order to achieve bandwidth efficiency. This concept is the basis for rate distortion theory, that is, receivers might tolerate some visual distortion in exchange for bandwidth conservation.

\subsection{Encoder [10]}

An entropy encoder further compresses the quantized values losslessly to give better overall compression. It uses a model to accurately determine the probabilities for each quantized value and produces an appropriate code based on these probabilities so that the resultant output code stream will be smaller than the input stream. The most commonly used entropy encoders are the Huffman encoder and the arithmetic encoder, although for applications requiring fast execution, simple run-length encoding (RLE) has proven very effective.

\subsection{F-IDCT Encode Integrating Out Edge Information [7]}

The posterior distribution of a segmentation of a portal image, given by the Gibbs distribution in [7], is first simplified by integrating out the edges. The edges have been integrated out; an estimate of the edges will be obtained as a by-product of the optimization process.

\section{EXPERIMENTAL RESULT}

The experimentation about the proposed method has been done. Here the FDCT transform coding applied on test image compression with Sinusoidal Noise Removal Method. Then system implemented FDCT and F-IDCT. Now for checking clarity, quality, and performances of transforms applied on each image process done evaluation between actual image (input image) and compressed image using PSNR and MSE values for each transforms. Following are sample compressed images, the compressed by FDCT and F-IDCT. In this original image is compressed and reconstructed system is getting the quality output image.

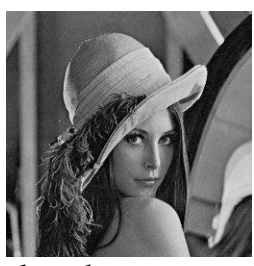

lena.bmp

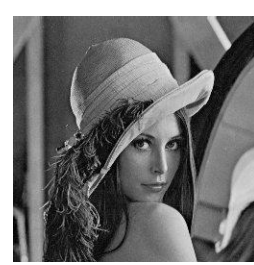

lena.tif

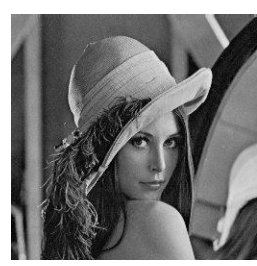

lena.png
Fig 6: Sample set of Original Image

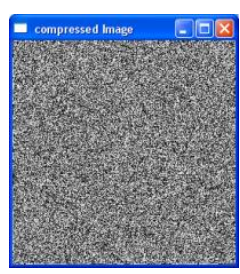

lena.bmp

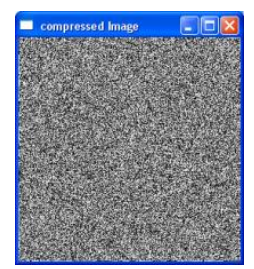

lena.tif

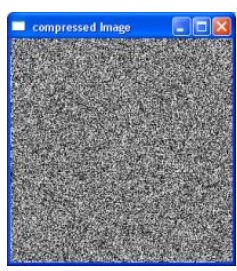

lena.png
Fig 7: Sample set of Compressed Image 


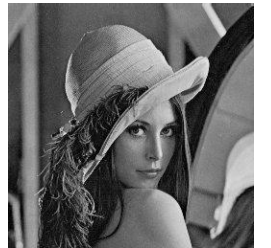

lena.bmp

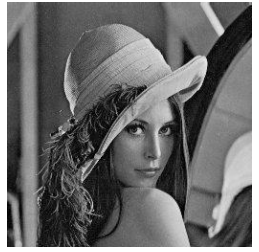

lena.tif

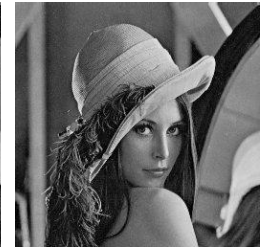

lena.png
Fig 8: Sample set of Reconstructed Image

Table.2: PSNR and MSE value

\begin{tabular}{|c|l|l|l|l|}
\hline \multirow{2}{*}{$\begin{array}{c}\text { Image } \\
\text { Type }\end{array}$} & \multicolumn{2}{|c|}{ PSNR } & \multicolumn{2}{c|}{ MSE } \\
\cline { 2 - 5 } & Existing & Proposed & Existing & Proposed \\
\hline lena.bmp & 42.0157 & 42.945 & 4.1019 & 4.09 \\
\hline lena.tif & 38.0163 & 38.6947 & 9.6985 & 8.27 \\
\hline lena.png & 47.063 & 47.425 & 1.122 & 1.087 \\
\hline
\end{tabular}

The Experimentation of the image compression is displayed above. The result of the image compression by the proposed method in compressed and decompressed the color image. The proposed method is getting the result as compared to existing method. The PSNR and MSE value consuming is structure of color image compression.

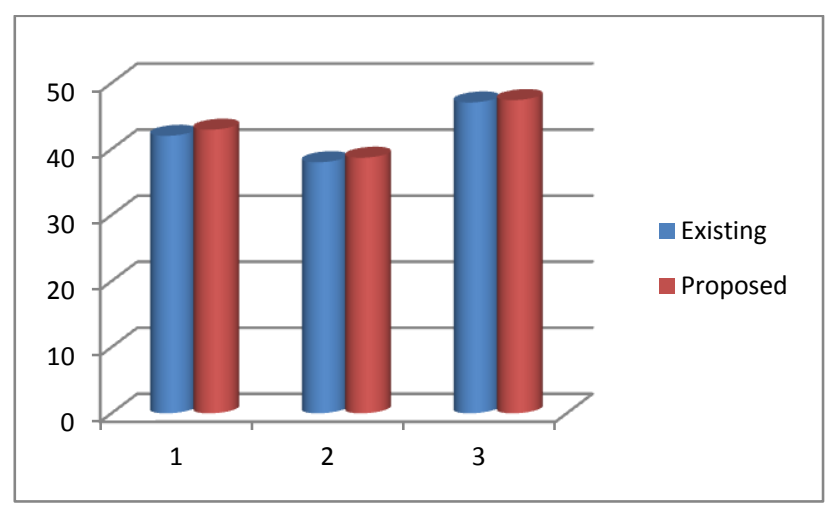

Fig 9: PSNR Representation

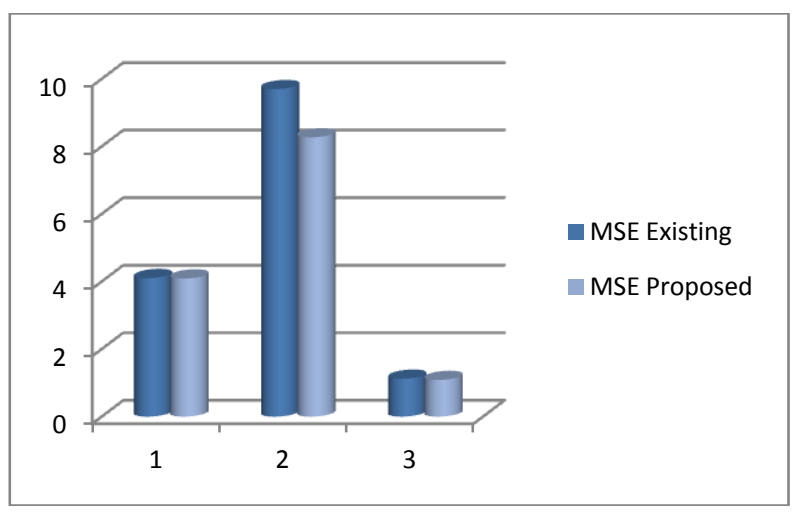

Fig 10: MSE Representation
In graphical representation system is calculating the building PSNR and MSE value of image compression comparison of existing with proposed system. In this existing system PSNR and MSE value is newly proposed algorithm is considerably reduced.

\section{RESULT AND DISCUSSION}

Different image formats like bmp, png and tif are used for testing and their PSNR and MSE are compared. The newly proposed method has achieved greater compression than that of existing method. The quality of reconstructed image is measured using parameters: MSE and PSNR. Table 1 reveals that the measurement of MSE and PSNR for newly proposed method is acceptable. The system observes that, the newly proposed method is much lesser than that of existing method. In this newly proposed method greater beneficial in terms of saving storage, reducing the image noise as well as effective reconstructed the color image. The quality of image is measured in terms of MSE and PSNR value. It is equally important to conserve the important uniqueness of an image after compression and reconstruction.

\section{CONCLUSION}

In this paper, system proposed reduces the noise in color image compression. Experimentation has been carried out on different image format successfully. The newly proposed method is simple. A good compression has been achieved with good PSNR and MSE.

The image performance based error free image compression in sinusoidal noise removal with Fine DCT and F-IDCT proposed here gives better quality of compression and reconstruct image. Here the Fine transform technique is used to best compression mechanism. This transform has shown around 50\% of improvement in time of compressed image as compared of existing one. The FDCT transform have shown around $70 \%$ of improvement in the time of compressed image and reconstruct image.

\section{REFERENCES}

[1] Sudeep D.Thepade, Shwetali Erandole, "Extended Performance Comparison Of Filing Based Image Compression Using Wavelet Transform And Hybrid Wavelet Transforms", Proc of 2013 IEEE Conf on Information and Communication Technologies, IEEE 2013.

[2] H.B.Kakre, A.Athawale, P.N.Halarnkar, V.K. Banura, "Performance Comparison of DCT and Walsh Transform For Steganography”, ACM 2010.

[3] S.Parveen Banu, Dr.Y.Venkataramani, "An Efficient Hybrid Image Compression Scheme based on Correlation of Pixels for Storage and Transmission of Images", International Journal of Computer Application. Volume 18- No.3, March 2011.

[4] M. Nelson and 1. L.Gailly, The Data Compression Book, 2nd ed. New York: M \& T Books, 1996.

[5] A K.Jain, "Image data compression: A review," Proc.IEEE, vol. 69,pp. 349-389, 1981.

[6] R.C.Gonzales and P. Wintz, Digital Image Processing,3rd ed. Reading, MA: Addison-Wesley, 1992.

[7] Neelam K. Patil, Suresh F.Murgod, Lokesh Boregowda, V.R.Udupi, "Adaptive Texture and Color Feature Based Color Image Compression”, Proc ICSS 2013, Mar 13. 
[8] Daidi Zhong, Irek.Defee , "Pattern Recognition by Grouping Areas in OCT Compressed Images", Proceedings of the 6th Nordic Signal Processing Symposium - NORSIG 2004 June 9 - 11, 2004, Espoo, Finland.

[9] Yung-Gi Wu, "Medical Image Compression by Sampling DCT Coefficients",IEEE Transactions on Information Technology in Biomedicine, VOL. 6, NO. 1, MARCH 2002.

[10] Racape F., Lefort, S.; Francois, E.; Babel, M. ; Deforges, o."Adaptive pixel/patch-based synthesis for texture compression", Image Processing (ICIP), 2011 18th IEEE International Conference on 11-14 Sept. 2011.

[11] S.C.Tai, Y.G.Wu and C.W.Lin, "An adaptive 3-D discrete cosine transform coder for medical image compression, " IEEE Trans. Inform. Tech. Biomed., vol. 4, pp. 259-263, 2000.

[12] M.M. Siddeq; M.A.Radrigues, “A Novel Image Compression Algorithm for High Resolution 3D Reconstruction", Springer 26 Mar2014.

[13] Sukho Lee, Sang-Wook Park, Paul Oh, and Moon Gi Kang, "Colorization-Based Compression Using Optimization", IEEE Trans. On Image Processing, Vol.22, No.7, July2013.
[14] Rafael C. Gonzalez, Rchard E. Woods, "Digital Image Processing", Third Edition.

\section{AUTHOR PROFILE}

Dr.G.J.Joyce Mary completed her Ph.D in the area of Parallel Computing in 2012. Now she is working as a Research supervisor and Associate Professor in the Department of Computer Science and Engineering, PRIST University, Thanjavur, Tamilnadu, India. Her area of interest includes Parallel Computing, Digital Image Processing and Webservice.

A.Ramachandran received the M.Tech degree in Computer and Information Technology from Department of Information Technology and Engineering at Manonmaniam Sundaranar University, Tirunelvelli, Tamilnadu, India. He was a Research Scholar in the Department of Computer Science and Engineering, Center for Research and Development, PRIST University, Vallam, Thanjavur, Tamilnadu, India. He was working as Assistant Professor in the Department of Computer Science and Engineering, Srinivasan Engineering College, Perambalur, Tamilnadu, India. His current research interest includes image segmentation, image compression, image reconstruction, image noise removal, image restoration and enhancement. 